\title{
Synchronization in a goal-directed task: human movement coordination with each other and robotic partners
}

\author{
Tamara Lorenz, Alexander Mörtl, Björn Vlaskamp, Anna Schubö, Sandra Hirche
}

\begin{abstract}
Synchronization occurs frequently in human behaviour: Everybody has experienced that in a group of people walking pace tends to equalize. The phenomenon of synchrony has been established in the literature in tasks which have little in common with daily life such as pendulum swinging and chair rocking. We extend the knowledge about human movement synchronization by showing that it also occurs during goal-directed actions. In a first experiment, we investigate how synchrony emerges develops over time. In a second experiment, we show that humans also synchronize their actions with a robot. Results are interpreted in the light of joint action theory. Possible implications and improvements for human-robot interaction are discussed.
\end{abstract}

\section{INTRODUCTION}

$\mathrm{D}$ URING human-robot interaction it is important that humans can coordinate their movements with the $\operatorname{robot}(s)$ in a confident and safe way. One approach to achieve this is to investigate how humans interact with each other, to extract general interaction principles from that and to implement robot behaviour in accordance with those principles [1]. However, the study of human interaction is complex and only very few interaction principles have been uncovered. In this light, an interesting phenomenon is that people tend to synchronize their actions. For example when walking in a group, people tend to synchronize gait [2]. Similar synchronization of behaviour has been observed in several circumstances and tasks such as swinging handheld pendulums [3], rocking in chairs [4] or moving one's legs [5]. Synchronization appears to be an integrated part of human interaction. It enhances perceptual sensitivity and thereby increases success in a joint action task [6]. In social psychology synchronization is furthermore acknowledged to lead to the attribution of more positive characteristics to an opponent [7] and is known to increase rapport in different situations of daily life such as teacher-student relation [8] and mother-child-bonding [9].

Synchronization seems to be an essential part of our behaviour and additionally provides a relatively easy way

Manuscript received March 17, 2011. This work was supported by the German Research Foundation (DFG) as part of the Excellence Cluster "Cognition for Technical Systems" (CoTeSys, Project \#410).

T. Lorenz (+49-(0)89-289-25735; tamara.lorenz@lmu.de) and B. Vlaskamp are with the Experimental Psychology Institute, LudwigMaximilians University, Munich, Germany.

A. Mörtl und S. Hirche are with the Institute of Automatic Control Engineering, Technische Universität München, Munich, Germany

A. Schubö is with the Psychology Section, Philipps University Marburg, Germany. of describing human behaviour quantitatively. Considering that, synchronizing one's movements may provide an excellent opportunity to improve human robot interaction and thus the way robots help people achieve certain manipulations of the environment. These manipulations can involve cleaning up the house, doing the dishes or serving food. What all these tasks have in common is that they require planning and precision of movements and therefore joint action partners should be tuned to each other's movements. However, until now synchronization has only been investigated in very specific circumstances as those described above. It is thus unclear whether synchronization would occur in tasks of daily life and also the applicability of these findings for human-robot interaction remains questionable.

In the present study we asked if synchronization also occurs in a task requiring goal-directed movements. Our participants carried a pen from a start point to a target point and back again while their interaction partners were sitting at the opposite side of the table and executed the same task. The performed action bears similarities to everyday tasks that require picking or placing of objects in a shared workspace such as cleaning or setting up the table, setting up a game of chess, etc.: namely goal directed and precise movements in a shared workspace.

Additionally, we enquire if people would synchronize their movements with robots in the same task. If so, we have evidence for a general principle for interaction that is relatively easy to implement in robots and may enhance the interaction experience for the human partner.

\section{METHOD}

\section{A. Participants}

In total 10 dyads (13 male, 7 female) participated in the human interaction experiment (age: 18 to 28 years $(\mathrm{M}=23.4)$. In the human-robot interaction experiment, six people (4 male, 2 female) participated (age: 20-28 years, $M=23$ ). All participants were right-handed.

\section{B. Experimental Setup}

Two experiments were done: (1) human-human interaction (HHI) in which two participants were interacting with each other; (2) human-robot interaction (HRI) in which the participant was interacting with a robot. In both experiments agents were sitting at a round table $(\mathrm{r}=0.575 \mathrm{~m})$ facing each other, see Fig. 1. Four coloured dots with a diameter of $8 \mathrm{~mm}$ were marked at the table. Each dot was surrounded by a white area (diameter 


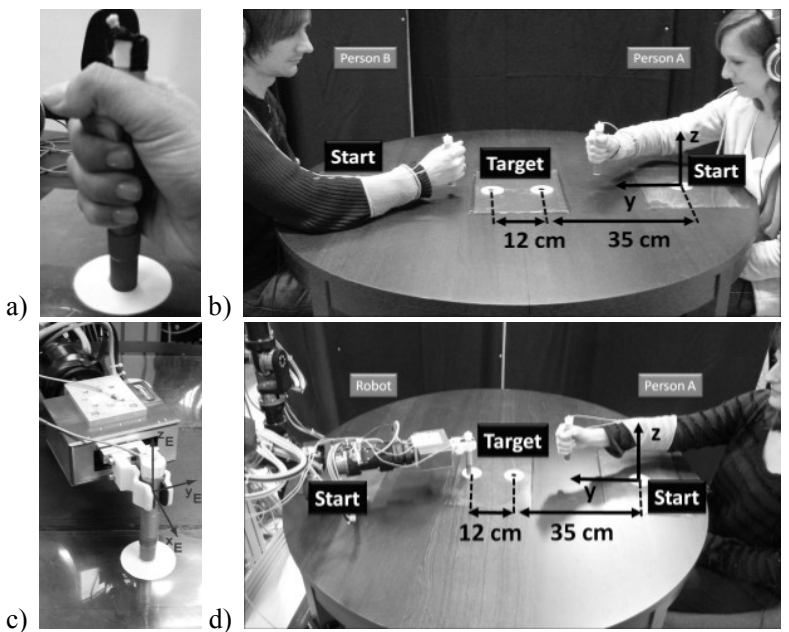

Fig. 1. Experimental setup for both experiments: a) human hand holding thepen; b) setup for human interaction (HHI) c) robotic gripper holding the pen; c) setup for human-robot interaction (HRI). LEDs for motion tracking are attached to the top of the pen.

$60 \mathrm{~mm})$. Agents were assigned two dots of the same colour at the respective side of the table. The dot which was located closer to the body was defined to be the start, the other the target. Participants were each equipped with a pen and instructed to grab the pen making a fist around it with their right hand. Additionally they had to wear SONY stereo headphones (MDR-XD200) with a connection to the control PC. Movements were recorded using an infrared tracking system (PTI Visualeyez II VZ4000). Wired LEDs were attached to the top of each pen and the thumb of agents' right hands/ the robot's gripper (see Fig. 1a, c). LEDs were tracked with a camera bar mounted at the ceiling. The tracking system had an online sampling rate of $30 \mathrm{~Hz}$ used for calculating the start signal delays in condition (2) and (3), see section II.B, and an offline sampling rate of $200 \mathrm{~Hz}$ used for data recording.

\section{A. Robotic Partner}

The HRI experiment was done with a human-size mobile robot with two 7 degrees-of-freedom arms [10]. We assigned a fixed position to the robot relative to the table in which it was able to reach the respective start and target areas. Motion of the robot was restricted to movements of its right arm during the experiment. In order to grasp the pen, we equipped the robot with a twofinger gripper. The robot grasped the pen in a predefined stable grasping position and did not release it during the experiment, see Fig. 1. Endpoint movement trajectories of the robot arm were composed from fifth-order polynomial segments yielding human-like minimum-jerk movements [11]. We analyzed the recorded human trajectories from the HHI experiment and reproduced the following varying features to make the movements even more human-like: variance of hitting precision in the start and target area, maximum elevation above the table including its variance and relative position between start and target area, maximum deflection from the straight line taken in the table plane, see Fig. 2. Four trajectory

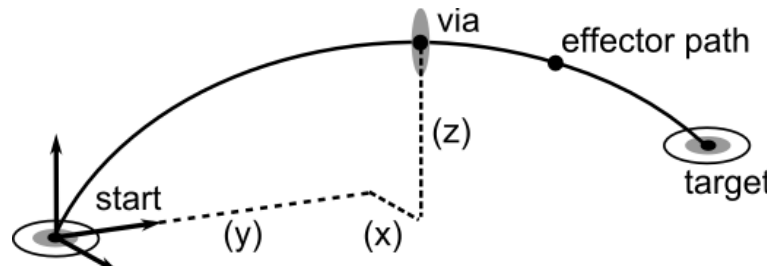

Fig. 2. Sample effector path composed by two fifth-order polynomials interpolating between start, via point and target point. The features maximum deflection in the table plane (x), relative position between start and target (y) and maximum elevation above the table $(\mathrm{z})$ specify the via point. Shaded areas indicate modeled variances.

segments per movement cycle were fitted to model these features, yielding two end-points and two via-points per Cartesian direction respectively. The robot's arm trajectory was composed by interpolation of two fifth order polynomials, one between start- and via-point, the other from via- to target-point and reverse. Interpolation was done separately in the three Cartesian directions similar to Huber et al. [12]. The orientation of the robot arm was commanded such that the $\mathrm{z}_{\mathrm{E}}$-axis of the arm's endpoint always pointed upwards normal to the table plane (see Fig. 1c). The grippers orientation angle around the $\mathrm{Z}_{\mathrm{E}}$-axis had to be changed between start and target area to fit the workspace limitations of the robot arm. Due to technical and safety reasons the per-cycle frequency of the robot's movements was set to $0.56 \mathrm{~Hz}$.

\section{B. Task and Procedure}

Participants were instructed to rest in the starting position with their pen upwards until they heard an acoustic signal through their headphones. This was the signal to start moving the pen from their individual start position to the respective target position, tap on the dot, move back and tap on the start position. Moving forward, tapping, moving backward and tapping again was defined as one cycle. Cycles were to be repeated until a second acoustic signal was presented which was automatically triggered when both agents had performed at least 10 cycles. Note that participants were naïve as to the goal of the experiment and were not instructed to adapt their movements to each other.

When people are working together in daily life they might not start their tasks in sync. One of the agents might already have preceded a certain part of the required trajectory before the second agent starts to move. To emulate these circumstances, different timings of start signals were given. In both experiments (HHI/HRI) three different start conditions were introduced: (1) zerodistance: the start signal was presented at the same time for both participants; (2) half-distance: the start signal for the second person was presented when the first person passed half the way to the target; (3) full-distance: the start signal for the second person was presented when the first person had reached the target.

Both experiments were performed in six sets which consisted of six trials with equal start condition. This led to a total of 12 repetitions for each condition. During the conditions (2) and (3) the first start signal was assigned 


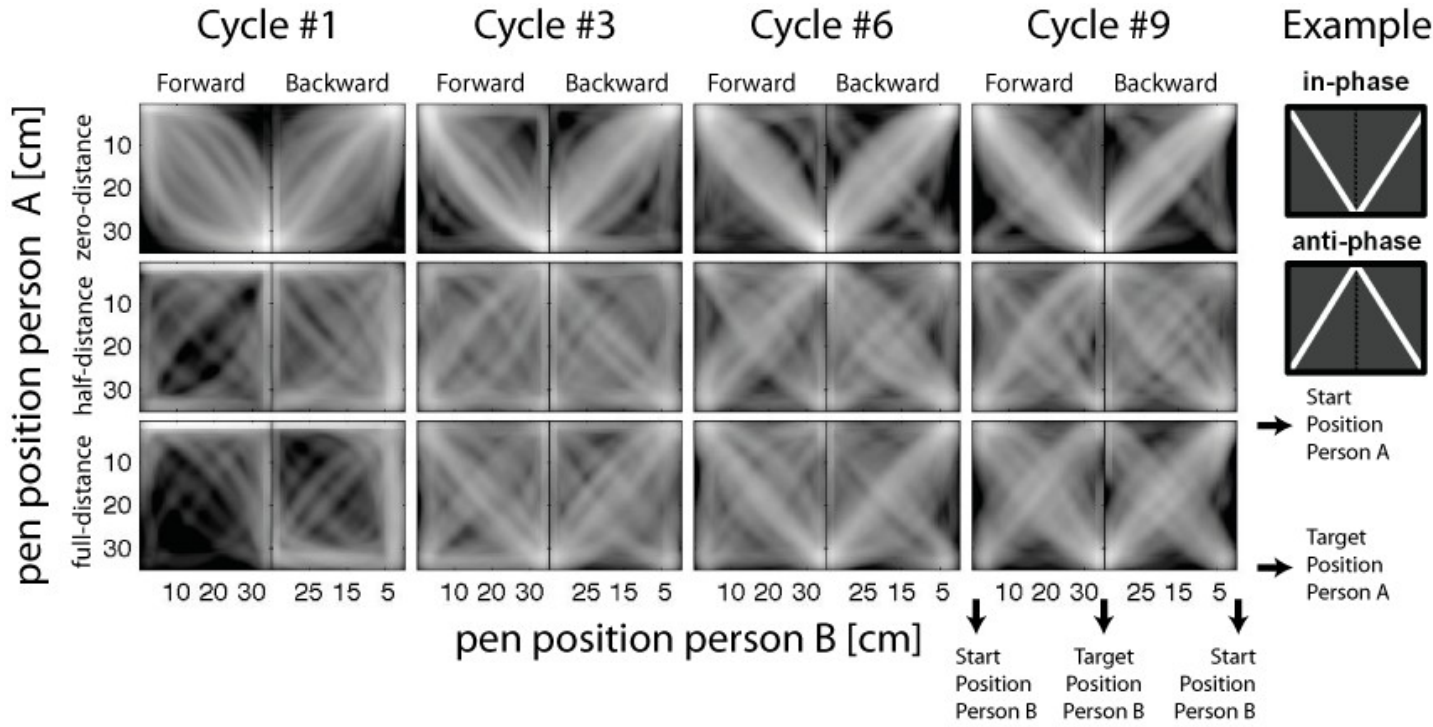

Fig. 3. Frequency distribution of relative movement data in action direction (y) for the conditions zero-distance (upper panels), half-distance (middle panels) and full-distance (lower panels); forward and backward movements are plotted separately for cycle number 1,3,6 and 9; the bright lines show the frequency of the relative position of person B (ordinate) towards A (abscissa); in the zero-distance condition the lines become straighter which shows that the frequency of being at the same place at the same time (in-phase) is increasing towards the $9^{\text {th }}$ cycle; this indicates that people are in fact adapting their movements to each other. For half-distance and full-distance it can additionally be observed that the interaction partner is at the opposite place at the same time (anti-phase), which is more expressed if people start with fulldistance and in general later than in the zero-distance condition; in the upper right an example of perfect in-phase/anti-phase relation is given.

randomly to one of both participants and counterbalanced in each set. Note that the task did not require synchronization which allowed us to explore if it emerges naturally.

\section{Data Analysis}

Movement data of both experiments were processed in the same way: velocity was calculated from the Euclidian distance between data points in Cartesian coordinates. A fourth order low-pass Butterworth filter with a cut-off frequency of $10 \mathrm{~Hz}$ was applied. The resulting phase shift was corrected by applying the same filter reversely. Every time the velocity-time profile had a minimum, a pen tapping was assumed. Herewith the data was divided into 10 cycles. Due to variations in trial terminations, the $10^{\text {th }}$ cycle was excluded from analysis.

Using one of the methods tested by Teasdale et al. [13], movement onset was calculated as the first time the movement crossed $15 \%$ of the peak acceleration of the trial.

\section{RESULTS}

\section{A. Human-Human Interaction}

First, we looked at the relative positions of the dyads' hands. From literature it is known that during human synchronization of movements mainly two dominant states emerge: in-phase, i.e. agents execute the same part of the movement at the same time, or anti-phase, i.e. agents execute the opposite part of the movement at the same time [14]. In order to find out whether and how often a similar relation occurred in our experiment, we plotted a frequency distribution of all the movement data of person $\mathrm{A}$ as a function of the movement data of person B. For trials and cycles the y-position of person A was plotted as a function of person B's y-position, see also Fig. 2. The resulting curve was sampled with an underlying grid of $100 \times 100$ cells and the number of times each cell in the grid was hit by a curve was determined. Resulting frequencies were plotted as a heat map in which brightness codes frequency, see Fig. 3. In these plots, perfect synchrony appears as straight lines. When the participants are perfectly in-phase during forward movement, a line goes from top left to bottom right. During backwards movements - where the abscissa labelling goes down again, the plot shows a straight line from the bottom left to the top right. In perfect anti-phase the pattern is mirrored. When participants are out of sync, data appear curved. Going from left to right in Fig. 3, we can see that during the first cycle the data tend to be curved. This is particularly true in the half-distance and full-distance conditions. Here behaviour is very idiosyncratic, because very few straight lines become apparent. After cycle 1, people quickly adapt and by the time they enter cycle 3 , there are still curved lines, but straight lines have become more prevalent with possibly slightly more lines indicating in-phase movements. Hence, after only three cycles synchronization is established.

To scrutinize synchronization further, we analyzed the lag between the actions of each dyad, i.e. the betweendyad time difference in the execution of movements. We operationalize synchrony as the degree to which lag varies over time: the less lag varies, the more the actions are synchronized. Note that this means that also actions that are not executed at the same time can be executed synchronously. We calculated lag at several reference points within the action cycles: the target area entry, target area exit, start area entry, start area exit. Lag variability is calculated as the absolute difference at the 
reference point between two subsequent cycles. Because some cycles are executed faster than others we normalized lag to each cycle's duration (calculated as the average of both participants). In Fig. 4, lag variability is plotted as a function of the cycle difference for which it is calculated. The plotted value is the across-subject mean of within-subject median lag variability. The reference point for this figure was target area entry. Clearly, lag variability goes down as the task proceeds, $\mathrm{F}(7,63)=31.99, p<.001$. Contrasts reveal that the first, $\mathrm{F}(1,9)=58.03, p<.001$, and the second lag variability, $\mathrm{F}(1,8)=19.72, p<.01$, are significantly higher than the eighth, indicating that lag variability between time points in which people enter the target area is decreasing in the first cycles. No differences were observed for all other levels, all $p>$.1. We confirmed this finding by measuring lag variability at other reference points as well (target area exit, start area entry, start area exit) with similar results. So, clearly people adjusted their behaviour towards synchronized movements and they did so mostly in the beginning.

Since synchronization measured by lag variability occurred more often as more cycles had been executed, participants adapted their behaviour. Here different strategies could have been applied: participants could either have changed how long they dwelled in the start and target area, or they could have changed the speed at which they performed the movements and thus the movement time for this segment. Therefore we calculated dwell times and movement times and determined the difference regarding these measures between interaction partners. Because we know that synchronization improves over time, we expect either one of these measures or both to go down. Fig. 5 shows the dwell time differences in target and start area and forward/backward movement time differences between participants. A significant effect for cycle number was found for between-subject movement time differences during forward movements, $\mathrm{F}(8,72)=4.31, p<.001$. Contrasts show that movement

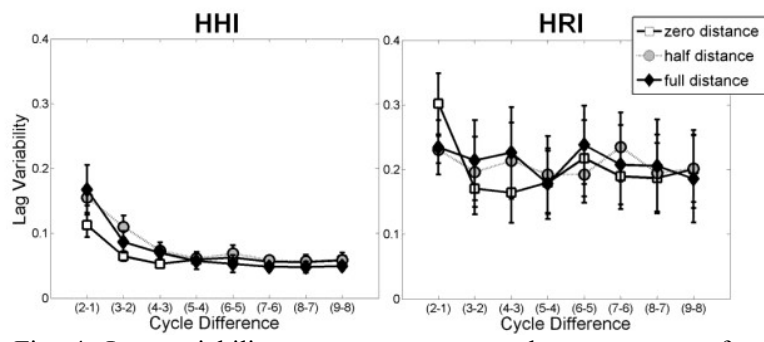

Fig. 4. Lag variability at target area entry between agents for human interaction (left panel) and human-robot interaction (right panel). The left panel shows that people adapt their movements towards a more stable pattern.

time differences were higher during the first forward movement compared to the last, $\mathrm{F}(1,9)=10.05, p<0.05$, whereas no differences were observed for the other levels, all $p>.09$. During backwards movements, no differences were observed, $p>$.6. So, only forward movement time differences seem to contribute substantially to synchronization.

Looking at differences in dwell times in the target area, there was a significant main effect for cycles, $F(8,72)=$ $12.70, p<.001$. Contrasts show that the difference in time spent in the target area during the first cycle was higher than in the last, $\mathrm{F}(1,9)=17.00, p<.01$. Also during the second cycle dwell time differences in the target area were higher than in the last cycle, $\mathrm{F}(1,9)=5.10, p=0.05$. For the other cycles no differences in dwell time were observed compared to the last cycle, all $p>$.3. Dwell time differences in the start area also showed a main effect of cycle $\mathrm{F}(8,72)=4.65, p<.001$. Contrasts reveal a difference between the first and the last cycle, $F(1,9)=$ $16.87, p<.01$. For all other cycles no differences were found to the last one, all $p>.1$. Regarding the factor condition, no effect was observed for all comparisons, $p>$ .1. Also no interaction effects were observed, $p>$.1. In short, dwell times convincingly reflect synchronization in the course of a trial.

We also had a look on the average frequency with which the human movements were executed. Taking the
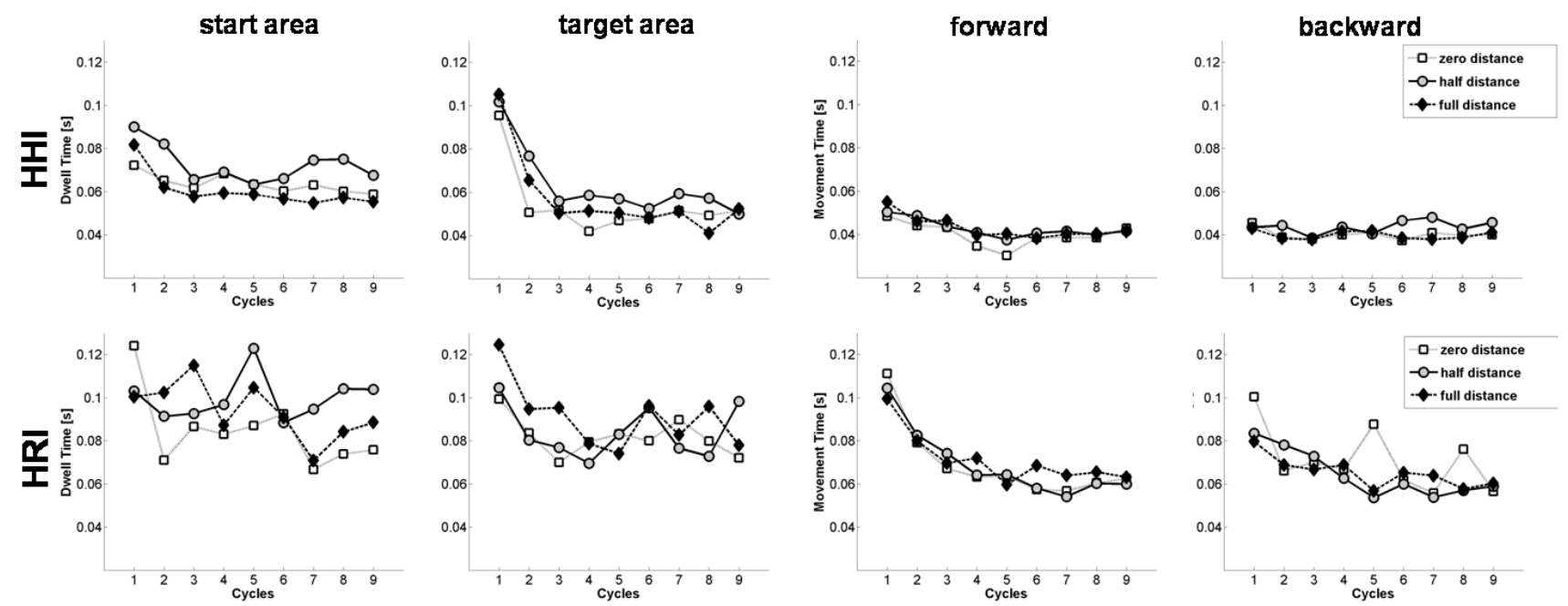

Fig. 5. Dwell time differences averaged over dyads in start and target area (left panels) and movement time differences during forward and backward movements (right panels); upper graphs show the data obtained from the human interaction experiment; lower graphs data from the human-robot interaction task; 
mean over all participants we found that humans performed with a frequency of $0.73 \mathrm{~Hz}$.

\section{B. Human-Robot Interaction}

Now that we found synchronization in goal-directed human-human interaction tasks, an interesting question is whether this phenomenon also occurs in human-robot interaction. In order to determine this, we applied identical measures used for analyzing the HHI experiment to analyze human-robot interaction.

During HRI, two participants were roughly moving at twice the rate of the maximal robot frequency of $0.56 \mathrm{~Hz}$ throughout the whole session. Although they were possibly adapting to the robot using a $2: 1$ rhythm, their data was not included into statistical analysis. From the remaining data 22 trials had to be excluded due to sensor malfunction. They were roughly equally distributed over conditions. Note that due to the small sample size, there may not be enough statistical power to reveal effects yet.

Nevertheless, we found initial evidence for synchronization. For the forward movement a main effect of movement time difference was found over cycles, $\mathrm{F}(8,24)=11.86, p<.001$. Contrasts reveal a difference between the first and the last cycle, $F(1,3)=37.67$, $p<0.01$, and between the third and the last cycle $\mathrm{F}(1,3)=11.14, p<0.05$. During backwards movement there is also a main effect of cycles, $\mathrm{F}(8,24)=3.81$, $p<0.01$, with a significant difference between the first and the last, $\mathrm{F}(1,3)=10.98, p<0.05$, and the third and the last cycle, $\mathrm{F}(1,3)=11.41, p<0.05$. Dwell time differences didn't show a main effect for the factor cycles in neither the target area, $p>.4$, nor the start area $p>.2$. Similar to the human interaction data, within all comparisons we didn't find any significant effect for the factor condition, all $p>.4$, nor any significant interaction effect, all $p>.1$. Watching Fig. 4, a decrease in lag variability of entry to target area across cycle differences seems evident; however there was no statistical effect, $p>.2$.

\section{DISCUSSION}

It is known that people tend to synchronize their movements in simple rhythmical and repetitive tasks like pendulum swinging [3], or rocking in chairs [4]. With the study at hand we answer the question if people also synchronize their movements in a goal-directed task. Our findings suggest that they do so and they do so quickly.

\section{A. Human Synchronization Strategy in a Goal- Directed Task}

In our HHI experiment, the results between conditions are very similar, but certainly the frequency distributions indicate that there are some differences.

Firstly, if people started moving at zero-distance they showed a clear tendency towards a stable synchronization pattern. The more cycles were performed, the more people were at the same time in the same part of their movement.
Secondly, in the full-distance and in the half-distance condition we see that in most trials stable synchronization is achieved, but this pattern is established later than in the zero-distance condition.

Thirdly, in the full-distance condition anti-phase and in-phase relationships occur roughly equally often which was also frequently observed in the half-distance condition. One might have expected that if zero-distance mainly leads to an in-phase relation, full-distance would mainly lead to an anti-phase relation. Altogether these results show that people synchronize their movements in goal directed tasks and it also shows that the movements are continuously adapted.

In the study of Richardson et al. [4] people were instructed to start moving at different times and it was found that during a non-goal directed task like rocking in chairs people nevertheless mainly exhibit in-phase relation independent from the temporal difference with which they started moving. It was argued that for the unintentional synchronization during rocking, the attractor anti-phase was too weak to sustain any significant coordination. In contrast, our results suggest that in a goal-directed task anti-phase is a frequently appearing attractor. It is possible that agents chose the attractor that is closest. For example it might be that in some cases the movement of the first person was performed like a single action because the workspace was still empty. If so then it would be performed slower than in joint action. Next, when the second person started moving, he/she entered a joint action task and may apply strategies other than those of the first person like leaving the target area more quickly [15]. The single action of one person in relation to the joint action strategy of the other person could lead to a decreased time difference which results again in an inphase relation as this is the closer attractor, so to say. Alternatively it could be that precisely hitting the target and the start point had a higher priority than synchronization because the latter was not required nor instructed. Only in cases in which an attractor was already close, synchronization was aspired over time.

In order to find out how adaptation emerges, we looked at the differences of dwell time in the target and start area and at the differences of movement time for the forward and backward movement. For the first forward movement we found a significantly larger temporal difference between interaction partners than in all other cycles. It seems plausible that in an interaction situation people slow down to see what the other person does. As gaze tends to precede manual movements [16] it would be easy to quickly glance at the activity of the other agent during forward movements (because the agents were sitting opposite of each other). By slowing down, resources could be freed-up and the own and the other's movement could be judged [17]. This seems especially plausible because no changes in movement time difference were found for the backwards movement.

Similar results were obtained for the difference in dwell time in the start and in the target area. During the first cycles the dwell time differences in target and start areas 
were higher than during the other cycles. This higher dwell time difference may indicate that one agent was waiting for the other agent. Once both agents are located in the same place, synchronous behaviour can easily be established by starting off synchronously again. That establishing the pattern seemed to take a bit longer for the full-distance condition might be due to the fact that the movement is harder to start off simultaneously from defined positions if these are further apart in space, namely one person rests in the target area while the other one rests in the start area.

The fact that no differences between conditions were found shows that the strategies applied to establish coordination are the same regardless of how the movement is started.

\section{B. Human Synchronization Strategy in a Goal- Directed Task with a Robot}

The second experiment provides a first insight into synchronization of human movements to a robot. On the basis of the present set of data which involved four human-robot dyads, there are already signs that synchronization occurs. The data generally show a similar pattern like that of human interaction. Synchronization can be assumed because especially for zero-distance the lag variability for the first cycle difference is about one half higher than the others. This is remarkable because the robot was moving about a third slower than humans on average did during HHI. That could potentially have made it more difficult to synchronize and the relative high lag variability compared to the HHI condition may be related to this. Nevertheless, apart from two dyads which might also have adapted in another rhythm, i.e. $2: 1$, we find that people adapted to the robot. Thus, they slowed down in order to synchronize with the robot.

Additionally, average movement time difference between the human agents and the robot for the forward movements were higher in the first three cycles. If it is true what was discussed for human interaction, namely that the reactions of the interaction partner are judged and integrated during the first forward movement, then here the judgement of the robot's movements may have required more time. It is also possible that humans tried to "encourage" the robot to follow their movements which caused a greater lag. And although the robot might not have reacted in the expected way, synchronization still appeared. This is especially remarkable because adaptation to the interaction partner was possible, but not required to fulfil the task.

\section{CONCLUSION}

In the study at hand we investigated synchronization of movements between two agents. Synchronization has been studied in various scenarios (chair rocking, pendulum swinging) but not in tasks that relate to daily life. For our study we therefore included one key aspect of tasks that people often do together, namely that they require goal-directed movements. We showed that also under these constraints synchronization occurred.
Considering our results we provide evidence that synchronization may occur in a wide range of everyday tasks. Furthermore we found, that people also apply the same strategies when interacting with a robot. This is a useful finding for human-centred robotics because synchronization principles are relatively easy to implement and might therefore be a reasonable step towards predictable and safe human-robot interaction.

\section{REFERENCES}

[1] W. Erlhagen and E. Bicho, "The dynamic neural field approach to cognitive robotics," Journal of Neural Engineering, vol. 3, 2006, pp. R36-54.

[2] N.R. van Ulzen, C.J.C. Lamoth, A. Daffertshofer, G.R. Semin, and P.J. Beek, "Characteristics of instructed and uninstructed interpersonal coordination while walking side-by-side," Neuroscience Letters, vol. 432, 2008, pp. 88-93.

[3] M.J. Richardson, K.L. Marsh, and R.C. Schmidt, "Effects of visual and verbal interaction on unintentional interpersonal coordination," Journal of Experimental Psychology. Human perception and performance, vol. 31, 2005, pp. 62-79.

[4] M.J. Richardson, K.L. Marsh, R.W. Isenhower, J.R.L. Goodman, and R.C. Schmidt, "Rocking together: dynamics of intentional and unintentional interpersonal coordination," Human Movement Science, vol. 26, 2007, pp. 845-859.

[5] R.C. Schmidt, C. Carello, and M.T. Turvey, "Phase transitions and critical fluctuations in the visual coordination of rhythmic movements between people," Journal of Experimental Psychology. Human Perception and Performance, vol. 16, 1990, pp. 227-247.

[6] P. Valdesolo, J. Ouyang, and D. DeSteno, "The rhythm of joint action: Synchrony promotes cooperative ability," Journal of Experimental Social Psychology, vol. 46, 2010, pp. 693-695.

[7] L.K. Miles, L.K. Nind, and C.N. Macrae, "The rhythm of rapport: Interpersonal synchrony and social perception," Journal of Experimental Social Psychology, vol. 45, 2009, pp. 585-589.

[8] F.J. Bernieri, "Coordinated movement and rapport in teacherstudent interactions," Journal of Nonverbal Behavior, vol. 12, 1988, pp. $120-138$.

[9] R.A. Isabella and J. Belsky, "Interactional synchrony and the origins of infant-mother attachment: a replication study," Child Development, vol. 62, 1991, pp. 373-384.

[10] B. Stanczyk, "Developement and control of an Anthropomorphic Telerobotic System," Technische Universität München, 2006.

[11] T. Flash and N. Hogan, "The coordination of arm movements: an experimentally confirmed mathematical model," Journal of Neuroscience, vol. 5, 1985, pp. 1688-1703.

[12] M. Huber, H. Radrich, C. Wendt, M. Rickert, A. Knoll, T. Brandt, and S. Glasauer, "Evaluation of a novel biologically inspired trajectory generator in human-robot interaction," IEEE RO-MAN, 2009, pp. 639-644.

[13] N. Teasdale, C. Bard, M. Fleury, D. Young, and L. Proteau, "Determining movement onsets from temporal series," Journal of Motor Behavior, vol. 25, 1993, pp. 97-106.

[14] H. Haken, J.A.S. Kelso, and H. Bunz, "A theoretical model of phase transitions in human hand movements," Biological Cybernetics, vol. 51, 1985, pp. 347-356.

[15] C. Vesper, A. Soutschek, and A. Schuboe, "Motion coordination affects movement parameters in a joint pick-and-place task," Quarterly Journal of Experimental Psychology (2006), vol. 62, 2009, pp. 2418-2432.

[16] R.S. Johansson, G. Westling, a Bäckström, and J.R. Flanagan, "Eye-hand coordination in object manipulation," The Journal of Neuroscience: the official Journal of the Society for Neuroscience, vol. 21, 2001, pp. 6917-6932.

[17] N. Sebanz, H. Bekkering, and G. Knoblich, "Joint action: bodies and minds moving together," Trends in Cognitive Sciences, vol. 10, 2006, pp. 70-76. 